\title{
ESTUDIO PILOTO DE LA PRUEBA DE PERCEPCIÓN MUSICAL CAMP EN IMPLANTADOS Y NORMOYENTES
}

Adelaida Plaza Ruiz MD*, Juan Manuel García MD**, Juan Carlos Izquierdo MD***

\section{Resumen}

En la búsqueda de una prueba de percepción musical estandarizada y aplicable en español, se evaluó el CAMP test creado en inglés por la Universidad de Washington para implantados y normoyentes. Objetivo: determinar las dificultades de su aplicación cuando el idioma nativo es español. Método: estudio de corte transversal con tres pruebas de percepción musical: umbral de tonos, percepción de melodías y timbre musical. Para correr el programa se tradujeron al español los comandos. Resultados: se reclutaron cinco pacientes, tres de ellos hombres (60\%). La edad mediana fue 42 años (RI de 40 a 43). El tiempo mediano de deprivación fue de 60 meses (RI 36 a 60). La mediana del tiempo de uso del implante fue de doce meses (RI 6 a 108). El resultado de detección del tono por frecuencias para el de $262 \mathrm{Htz}$ la mediana fue de 3.61 (RI 2.17 a 4.28), para $330 \mathrm{Htz}$ 3.72 (RI 2.94 a 4.28) y para $391 \mathrm{Htz} 5.5$ (RI 4.17 a 8.56). El porcentaje de reconocimiento con las melodías presentó una mediana de $8,33 \%$ (RI 5,56\% a 13,89\%) y el del timbre musical, medido por el reconocimiento de los instrumentos, 33,3\% (RI 20,83 a 45,83\%). Los controles fueron siete individuos sanos de los cuales cuatro eran mujeres (57,14\%), con edad mediana de 31 años (RI 18 a 44). En la percepción del tono el resultado de detección del umbral por frecuencias fue: para el de $262 \mathrm{Htz}$ de 1 (RI 0.67 a 3.39), para $330 \mathrm{Htz} 0.94$ (RI 0.5 a 4.39) y para $391 \mathrm{Htz} 0.72$ (RI 0.61 a 3.22). El porcentaje de reconocimiento con las melodías presentó una mediana de $69 \%$ (RI 33,3 a 83,3\%) y el del timbre musical 62,5\% (RI 45,8 a 75\%). La mayoría de los sujetos evaluados (10 de 12) realizaron la prueba sin ayuda de los examinadores, solo dos la requirieron para el manejo del programa, pero entendieron y pudieron completarla. De las tres características musicales evaluadas, la identificación de las melodías fue la más difícil. Conclusión: la prueba CAMP de percepción musical resulta útil fácil de aplicar en normoyentes y usuarios de implante coclear cuyo idioma nativo sea español, cuando se dispone de cartillas con traducción de los comandos.

Palabras clave: implantes cocleares, música, CAMP test.

Abreviaturas: IC, implante(s) coclear(es); RI, rango intercuartílico.

\section{CLINICAL ASSESMENT OF THE MUSIC PERCEPTION TEST CAMP IN IMPLANT USERS AND NORMAL- HEARING SUBJECTS: A PILOT STUDY}

\section{Abstract}

The CAMP test, created by the University of Washington for English-speaking implant users and normal-hearing subjects was evaluated in the search to provide a standardized music perception assessment for Spanish-speaking subjects. Objective: to determine administration difficulties when Spanish is the native language. Method: a cross-sectional study developed to examine three aspects of music perception: pitch discrimination threshold, melody recognition

Fecha recibido: agosto 26 de $201 \mathrm{l}$ - Fecha aceptado: diciembre 12 de $201 \mathrm{I}$.

* Especialista, residente de Otología, Fundación Universitaria de Ciencias de la Salud. Bogotá DC. Colombia.

** Coordinador del programa de Otología, Hospital de San José, Fundación Universitaria de Ciencias de la Salud. Bogotá DC. Colombia.
*** Médico otólogo, Hospital de San José. Bogotá DC. Colombia.

**** Licet Villamizar Gómez, Asesora metodológica. Profesora Asistente, División de Investigaciones, Fundación Universitaria de Ciencias de la Salud. Bogotá DC. Colombia. 
and timbre recognition. Commands were translated into Spanish in order to run the program. Results: Five subjects were recruited three of them men $(60 \%)$. The median age was 42 years (IR 40 to 43 ). The average time of auditory deprivation was 60 months (IR 36 to 60). The average time of implant use was 12 months (IR 6 to 108). The median for pitch discrimination by frequencies for $262 \mathrm{Htz}$ was 3.61 (IR 2.17 to 4.28 ), for $330 \mathrm{Htz} 3.72$ (IR 2.94 to 4.28 ) and for $391 \mathrm{Htz} 5.5$ (IR 4.17 to 8.56). The median for melody recognition was $8.33 \%$ (IR $5.56 \%$ to $13.89 \%$ ) and timbre recognition, measured by instrument recognition was $33.3 \%$ (IR $20.83 \%$ to 45.83 ). The control group included seven normal-hearing individuals, 4 were women (57.14\%) and mean age was 31 years (IR 18 to 44). In this control group, the median for pitch discrimination by frequencies for $262 \mathrm{Htz}$ was 1 (IR 0.67 to 3.39), for $330 \mathrm{Htz} 0.94$ (IR 0.5 to 4.39) and for $391 \mathrm{Htz} 0.72$ (IR 0.61 to 3.22). The median for melody recognition was $69 \%$ (IR $33.33 \%$ to $83.3 \%$ ) and timbre recognition was $62.5 \%$ (IR $45.8 \%$ to $75 \%$ ). The majority of individuals assessed (10 out of 12 ) self-administered the test with no help from the examiners, only two required some assistance to handle the program, but understood it and were able to complete the test. Of the 3 musical aspects evaluated, the most difficult was melody identification. Conclusions: The CAMP test for music perception is easily applied in Spanish-speaking normal-hearing individuals and cochlear implant users when instructions translated into Spanish are available.

Key words: cochlear implants, music, CAMP test.

\section{Introducción}

El objetivo del uso del IC desde su empleo exitoso por primera vez a finales de los años sesenta por William House, hace ya más de 50 años, ha pasado de querer solo mejorar la discriminación del lenguaje a desear brindar al paciente sordo la información auditiva necesaria para considerar su vida lo más normal posible. Siendo la música parte de un contexto sociocultural importante en cualquier individuo, es lógica la demanda de optimizar dicha información brindada por el implante.

El IC puede ofrecer mejoría importante del lenguaje alcanzando en promedio el $80 \%$ y aún más en pruebas de contexto cerrado. ${ }^{1-3}$ Diversos estudios muestran la dificultad de la percepción musical en aquellos con IC en especial al ser evaluados aspectos como el timbre y el tono. ${ }^{2,4}$ Sabemos que el disfrute musical conlleva la activación de vías que trascienden las áreas centrales solo auditivas y es clara la extensión a centros primitivos emocionales como la estría ventral, el tallo, amígdala, corteza orbitofrontal y prefrontal ventromedial $^{5}$, y cómo estas activaciones varían entre los individuos estudiados. Lo anterior conduce a los pacientes a obtener información y placer de las claves musicales transmitidas por su implante para mejorar su calidad de vida, en especial si la música ha sido parte fundamental de ella.
Los IC están diseñados para transmitir aspectos del sonido característicos del habla y existen varios códigos de estrategias para lograrlo. Los usuarios de IC han demostrado una increíble habilidad para percibir el habla, pero a pesar de estos logros los elementos básicos del área musical son un absoluto reto para la mayoría de los implantados. Muchos estudios no logran encontrar correlación o es muy débil entre las variables demográficas como edad, género, tipo de implante utilizado, estrategia de procesamiento de lenguaje o previo entrenamiento musical antes de la sordera. ${ }^{6}$

Por años hemos sabido que la especialización para el lenguaje es el hemisferio dominante izquierdo distribuyéndose en forma amplia alrededor de la cisura de Silvio en las áreas 21 y 42 de Broca y Wernicke, responsables de la producción y comprensión del lenguaje. Mientras que para la percepción musical los estudios soportan la noción de la especialización del hemisferio derecho para estas tareas, en especial del tono. ${ }^{7-9}$

Es claro, sin embargo, que no es solo una división de ambas habilidades en estos hemisferios sino que las comunicaciones interhemisféricas entre ambas juegan un rol importante. ${ }^{10,11}$ Son estas informaciones interhemisféricas y su función en la correlación de una y otra habilidades al ser evaluadas, las que pueden ser respon- 
sables del entrenamiento musical y su implicación en la mejoría del desempeño del lenguaje posimplante, lo que constituye la base de nuestra hipótesis de estudio.

La música se ha considerado siempre como un lenguaje universal accesible, independiente de las diferencias del hablado. Como una forma de comunicación abstracta la música goza de una libertad de entendimiento no compartida por los lenguajes tradicionales, cualquiera que él sea. La música en forma clara comparte con el lenguaje tradicional algunas características, las más importantes son: las de una jerarquía estructural (sintaxis/armonía), un vocabulario (palabras/acorde, intervalos), propiedades del tono (inflexión/timbre) y las claves temporales (prosodia/ritmo).

La música, así entendemos, es un concepto complejo que podemos reducir para facilitar su evaluación en las características principales de ritmo, tono, melodía y timbre. El ritmo depende en primer término de la codificación temporal y los pacientes implantados son capaces de distinguir patrones de ritmo casi tan bien como los normales. ${ }^{12,13}$

La percepción del tono musical en los IC depende de la localización de los electrodos y su contacto con la cóclea, como también de la frecuencia de la estimulación eléctrica. Esta última puede jugar el papel más importante para distinguir entre tonos, en especial en las frecuencias bajas. ${ }^{13,14}$

La melodía está compuesta por el ritmo y el tono, que a menudo incluyen un número de claves auditivas como la letra o patrones de ritmo, familiares para ayudar a los pacientes con IC. Estos son en general capaces de reconocer melodías con diferentes patrones de ritmo, pero si estas claves se remueven la tarea se vuelve más difícil. ${ }^{15}$

El timbre es definido como una combinación de cualidades del sonido que lo distingue de otros con el mismo tono y volumen, como lo hacemos con los producidos por diferentes instrumentos. ${ }^{16}$ Este depende de la información espectral y temporal y la mayoría de los pacientes con IC califican peor que los normoyentes para esta tarea. ${ }^{4,13,17}$
La discriminación del tono es una tarea de mayor reto para los implantados. ${ }^{4,18}$ Gefller y colaboradores ${ }^{4}$ encontraron que en ellos la habilidad para discriminar la dirección de tonos complejos requería un promedio de intervalo de umbral de 7.6 semitonos, mientras que los normales necesitaban solo 1.1 semitono. Además, este estudio demostró que la habilidad para discriminar el tono de las melodías se correlacionaba solo medianamente, sugiriendo que la discriminación del tono es necesaria pero no suficiente para hacerla con la melodía. La habilidad de la percepción del tono depende de la frecuencia evaluada. Clasificar un tono se refiere a la habilidad de señalar el cambio de dirección en forma correcta. Sin embargo, estos resultados dependen del tipo de IC utilizado, en especial electrodo largo versus corto, híbridos o estimulación eléctrica por los implantes.

Independiente del estímulo musical presentado o del componente musical presentados para que evalúen, los pacientes con IC se desempeñan peor que aquellos con audición normal en la discriminación del tono y en las tareas de reconocimiento de melodías y timbre musical (instrumentos). ${ }^{2,4,19}$

En nuestra investigación el principal marco de búsqueda de correlación entre percepción musical y desempeño del habla posimplante, uno de los mayores retos fue encontrar la prueba adecuada para valorar la percepción donde los componentes básicos de la música pudieran ser medidos, esto es tono, melodía y timbre musical. El primer inconveniente fue la falta de una prueba estándar en español para las instrucciones de las mediciones musicales y que fuera fácil de adquirir. Después de una ardua búsqueda encontramos que el CAMP test, creado por la Universidad de Washington en Iowa, cumplía con nuestros requerimientos, pero no sabíamos si las instrucciones podían ser fáciles de entender por normoyentes e implantados. Por esta razón decidimos desarrollar un ensayo piloto en ambas poblaciones tratando de mejorar la aplicación de la prueba musical.

Una de las ventajas encontradas en el CAMP test es que puede ser autoadministrado. ${ }^{16}$ Excepto por el uso de cartillas con traducción de los comandos al español 
para nuestro caso en particular, el paciente podría sin ayuda especializada, de forma individual, completar la prueba.

Este es el primer reporte de la serie de trabajos en curso en la Fundación Universitaria de Ciencias de la Salud sobre percepción musical en pacientes normales y con implante coclear.

\section{Materiales y métodos}

El ensayo piloto presenta un diseño de tipo corte transversal en el que se incluyen a pacientes normoyentes e implantados siendo su idioma nativo el español, en la que se evaluará la prueba musical CAMP test, en pro de detectar dificultades con el mismo que puedan ser corregidas antes de dar inicio al estudio de correlaciones del habla con percepción musical en pacientes implantados y normoyentes.

Los pacientes considerados casos eran adultos sordos poslinguales, mayores de 18 años y usuarios de su implante uni o bilateral por más de seis meses, remitidos por el servicio de audiología de los Hospitales de San José e Infantil Universitario de San José, que hubieran acudido a sus programaciones en forma regular y debían ser hablantes nativos de español.

Los controles fueron individuos voluntarios sin queja previa de audición, voluntarios, a quienes se aplicaron las mismas pruebas diagnósticas. Debían ser mayores de catorce años, que desearan participar en la prueba de percepción musical y fueran hablantes nativos en español. Se excluyeron para ambos grupos los sujetos ciegos o con alteraciones cognitivas evidentes.

Como instrumentos de medición se usaron el formulario de recolección de datos, el computador portátil marca HP con el software de CAMP test de percepción musical instalado y al que se conectó un parlante, de la marca JBL, para permitir la modificación adecuada del volumen si fuera necesario. Todos los profesionales participantes en el estudio recibieron entrenamiento previo para la aplicación de los instrumentos de medición y recolección de la información.
La fuente de datos fue directa con el paciente para los sociodemográficos y de tiempo de deprivación auditiva previa a su implante, cuya información y programación se tomaron de la audióloga y la historia clínica. El estudio incluyó cinco pacientes implantados con por lo menos seis meses de experiencia posimplante y siete personas normales. A todos se les informó sobre la naturaleza del estudio y se pidió su consentimiento informado para ser incluido.

La percepción musical fue evaluada usando un programa de computador CAMP test (clinical assessment of music perception) para pacientes con implante coclear. Este incluye grabaciones de alta fidelidad de instrumentos musicales sin sonidos sintetizados. Todos los estímulos musicales fueron presentados en campo abierto con el parlante al frente del sujeto a cero grados azimout y la interfase del computador a su alcance. A los sujetos de estudio se les permitió ajustar el volumen antes del inicio de la prueba hasta que se sintieran confortables con el mismo.

Se proporcionó al inicio una explicación clara de la prueba con sus tres etapas, brindando acceso a la cartilla explicativa con la traducción de los comandos a utilizar; no se permitió repetir la presentación ni decirles si su respuesta fue o no correcta. Los resultados se reportaron como umbrales de detección en la prueba de tono con su respectivo error estándar, y para melodía y ritmo como porcentajes correctos de cada uno.

\section{Prueba de tono}

En ambas pruebas de tono y melodía, se usaron tonos complejos sintetizados para proveer la frecuencia de base y de sobretono. Los tonos son creados en el CAMP test con una cubierta ("envelope") del espectro de una nota sola de piano sintético a un do medio (262 Hz), a la que se le aplicaron cubiertas (envelopes) temporales para evitar las claves de duración.

Debido a que la habilidad de la discriminación del tono se ha sugerido que depende de la frecuencia, esta prueba usa tres frecuencias base, la octava arriba de do, do 4 a $262 \mathrm{~Hz}$, mi 4 a $330 \mathrm{~Hz}$ y sol 4 a $391 \mathrm{~Hz}$. Aunque no representativo de todo el rango de tonos musicales, la 
prueba se limitó a estos por estar en la frecuencia vocal y musical más común en occidente, las más usadas en las melodías presentadas y para hacerla breve. ${ }^{15}$

Se presentaron dos tonos musicales (notas) y se pidió al sujeto investigado que señalara cuál era más agudo en la escala musical. Antes de la prueba verdadera el CAMP trae unos ejercicios previos de entrenamiento.

\section{Prueba de melodía}

La prueba de melodía incluye ocho canciones infantiles americanas y cuatro populares, siendo así doce melodías en total; siete muy conocidas entre los hispanohablantes y otras nada populares para nosotros. incluyen: Frére Jacques (panadero), Happy birthday (cumpleaños), Here comes the bride (marcha nupcial), Jingle bells (navidad, navidad), London bridge (el puente de Londres), Mary had a little lamb (Mary tenía una pequeña oveja), Old Mac Donalds (El viejo Mac Donalds), Rock a bye baby (duérmete niño), Row row your boat (rema, rema tu bote), Silent night (noche de paz), Three blind mice (tres ratones ciegos) y Twinkle twinkle little start (estrellita dónde estás).

Todas estas canciones fueron creadas en la octava alrededor y por encima de un do medio. Las melodías se tocan en forma isócrona, en que la línea incluye repeticiones de ocho notas. Esto con el fin de eliminar claves de ritmo que pueden confundir la identificación pura de la melodía. Cada una se crea usando un estándar de tiempo definido como un cuarto de nota (60 batidos por minuto), duración de la nota (50 mseg) y fue truncada a los ocho segundos para estandarizar la duración del clip musical. La amplitud de cada nota fue en forma aleatoria modificada $+/-4 \mathrm{dBs}$ para eliminar claves de volumen. ${ }^{15}$

Cada melodía fue ensayada dos veces antes de la prueba real como sería presentada en el CAMP test; si el paciente no realizaba el entrenamiento no podía continuar. Cada una se presentó tres veces en la prueba de percepción de melodías (en total 36). Una vez tocada el sujeto escogía en área aislada el nombre de la melodía escuchada de la lista que se le presentaba en la pantalla.

\section{Prueba de timbre}

La prueba subsecuencial de timbre incluyó ocho instrumentos musicales (cuerdas, metal, viento y percusión) tocando la secuencia de cinco notas acopladas a la octava arriba de do medio. Los instrumentos estaban representados por: percusión (piano), cuerda de nylon (guitarra), cuerda pura (violín y chelo), metal (trompeta) y viento (clarinete, flauta y saxofón). Los instrumentos se grabaron en vivo a 82 batidos por minuto a la misma intensidad de mezzo forte.

El paciente debió ensayar dos veces con cada uno de los instrumentos antes de poder continuar. Cada instrumento se presentó en la prueba tres veces (24 clips de instrumentos). Una vez presentados los acordes escogió en área aislada de la lista de instrumentos mostrados en la pantalla del computador.

\section{Análisis estadístico}

Se realizó un análisis descriptivo de las variables demográficas encontradas en ambos grupos estudiados. Las variables cualitativas se describieron con frecuencias absolutas y porcentajes, las cuantitativas se resumieron con medidas de tendencia central y de dispersión. La base de datos se construyó en Microsoft Excel® 2007. El análisis estadístico de la información se realizó con STATA versión 10.0.

\section{Resultados}

\section{Características demográficas: casos}

Para este estudio se tomaron cinco pacientes caso, de los cuales tres eran hombres $(60 \%)$. La edad mediana fue de 42 años (rango intercuartílico RI de 40 a 43) (Tabla 1). El tiempo mediano de deprivación auditiva previa a su implante (desde el momento de quedar sordo del todo hasta ser implantado) fue de 60 meses (RI 36 a 60). La mediana del tiempo de uso del implante fue de doce meses (RI 6 a 108). Tres pacientes tenían tipo Freedom, uno Nucleus 22 y otro Medel 
COMBI 40. Los cinco eran unilaterales, dos en oído derecho y tres en el izquierdo, uno de los casos era bimodal. Cuatro pacientes tenían procesador Freedom (100\%), tres con estrategia ACE y uno Speak, no tuvimos información sobre el procesador o estrategia del paciente usuario de Medel. El número de electrodos insertados fue de 18, 21, 22 y 23 en los cuatro casos que teníamos información. El paciente con 18 y 22 electrodos implantados tenía 11 y 16 activos. Ninguno de los implantados tenía previo entrenamiento musical formal. Tres consideraron que estaban de acuerdo en que disfrutaban la música y dos muy de acuerdo.

\section{Controles}

Los controles fueron siete individuos de los cuales cuatro eran mujeres $(57,14 \%)$. La edad mediana fue de 31 años (RI 18 a 44). Cuatro no tenían ningún tipo de entrenamiento musical, dos el básico de la escuela

\begin{tabular}{|c|c|c|c|}
\hline \multirow{2}{*}{ Características demográficas } & $\begin{array}{c}\text { Implantado } \\
\text { n (\%) }\end{array}$ & $\begin{array}{c}\text { Normoyente } \\
\text { n (\%) }\end{array}$ & \multirow{2}{*}{$\begin{array}{c}\text { TOTAL } \\
n=12(100)\end{array}$} \\
\hline & $\begin{array}{c}\text { Control } \\
n=7(58.33)\end{array}$ & $\begin{array}{c}\text { Casos } \\
n=5(41.67)\end{array}$ & \\
\hline Género & & & - \\
\hline masculino & $3(50.0)$ & $3(50.00)$ & $6(100)$ \\
\hline femenino & $4(66.67)$ & $2(33.33)$ & $6(100)$ \\
\hline Edad en años* & $31(18-44)$ & $42(40-43)$ & \\
\hline Tiempo de deprivación, en meses* & N/A & $60(36-60)$ & \\
\hline Tiempo de uso de su implante en meses * & N/A & $12(6-108)$ & \\
\hline \multicolumn{4}{|l|}{ Tipo de implante } \\
\hline Freedom & N/A & 4 & \\
\hline Nucleus 22 & N/A & 1 & \\
\hline Medel & & 1 & \\
\hline \multicolumn{4}{|l|}{ Tipo de procesador } \\
\hline Freedom & N/A & 4 & \\
\hline otros & & 1 & \\
\hline \multicolumn{4}{|l|}{ Estrategia de programa } \\
\hline$A C E$ & N/A & 4 & \\
\hline SPEAK & N/A & 1 & \\
\hline ¿? & N/A & 1 & \\
\hline Electrodos insertados \# & N/A & $20.75(1.8)$ & \\
\hline \multicolumn{4}{|l|}{ Entrenamiento musical previo } \\
\hline ninguno & 4 & 5 & \\
\hline entrenamiento básico & 2 & & \\
\hline en academia o con un instrumento & 1 & & \\
\hline \multicolumn{4}{|l|}{ Disfrute musical } \\
\hline \multicolumn{4}{|l|}{ nada } \\
\hline \multicolumn{4}{|l|}{ un poco } \\
\hline disfruta & 3 & 3 & \\
\hline disfruta mucho & 4 & 2 & \\
\hline
\end{tabular}

* Mediana (rango intercuartilico). 
y uno lo tuvo regular con clases de guitarra. Tres individuos consideraron que estaban de acuerdo en que disfrutaban la música y cuatro muy de acuerdo.

\section{Manejo técnico del CAMP test}

Ninguno de los pacientes tuvo dificultad con el uso de los comandos del programa. Uno con implante y otro normal refirieron bloqueo del sistema al pasar de tonos a melodías, siendo necesario después realizar solo el último para completar los puntajes de las tres pruebas sin tener que repetirlas todas.

Se ofreció ayuda en el manejo del programa si era necesario y solo dos de los doce participantes la solicitó. Fue fácil la autoadministración en ocho casos que contaban con mejor nivel educativo; el resto requirió soporte del profesional a cargo por dificultad en el manejo del computador o inseguridad en la mecánica de la prueba.

\section{Prueba de percepción musical en implantados}

En la percepción del tono el resultado del umbral mediano fue por frecuencias: para el tono de $262 \mathrm{Htz} 3.61$ (RI 2.17 a 4.28), para $330 \mathrm{Htz}$ fue de 3.72 (RI 2.94 a 4.28) y para $391 \mathrm{Htz} 5.5$ (RI 4.17 a 8.56 ) (Tablas 2, 3 y 4$)$.

El porcentaje de reconocimiento con las melodías presentó una mediana de 8,33\% (RI 5,56 a 13,89\%) y el del timbre musical, medido por el reconocimiento de los instrumentos, fue 33,3\% (RI 20,83 a 45,83\%). La duración total fue de 43 minutos (tono 16.65 , melodías 20.6 y timbre 6.17). Durante la prueba de tonos el implantado con menor número de electrodos activados (11), si la distancia en semitonos de las notas era muy corta, no identificó que eran dos sonidos y los interpretaba como uno solo; hallazgo no observado con el resto de implantados.

\begin{tabular}{|l|l|l|}
\hline \multicolumn{3}{|c|}{ Tabla 2. Percepción musical I2 participantes en la prueba piloto } \\
\hline \multicolumn{1}{|c|}{ Percepción musical } & \multicolumn{1}{c|}{ Implantado } & Normoyente \\
\cline { 2 - 3 } & \multicolumn{1}{|c|}{ Control $n=7$} & \multicolumn{1}{c|}{ Casos n=5 } \\
\hline Duración de la prueba en minutos* & 25.85 & 43 \\
\hline Duración de la prueba tonos * & 9.21 & 16.65 \\
\hline Duración de la prueba de melodia * & 12.49 & 20.6 \\
\hline Duración de la prueba timbre * & 4.52 & 6.17 \\
\hline 262 Htz mediana (RI) & $1(0.61-3.22)$ & $3.6(2.17-4.28)$ \\
\hline Umbral tono 330* & $0.94(0.5-4.39)$ & $3.72(2.94-4.28)$ \\
\hline Umbral tono 39I* & $0.72(0.61-3.22)$ & $5.5(4.17-8.56)$ \\
\hline Percepción melodía \% & & \\
\hline aciertos mediana (RI) & $69(33.3-83.3)$ & $8.33(5.56-13.89)$ \\
\hline Percepción timbre \% & & \\
\hline aciertos promedio (DS) & $61(79)$ & $33.3(20.83-45.83)$ \\
\hline
\end{tabular}

* Mediana 
Estudio piloto de la prueba de percepción musical CAMP en implantados y normoyentes

\begin{tabular}{|c|c|c|c|c|c|c|c|}
\hline Sujetos & Sujeto I & Sujeto 2 & Sujeto 3 & Sujeto 4 & Sujeto 5 & Sujeto 6 & Sujeto 7 \\
\hline Género & femenino & femenino & masculino & masculino & femenino & masculino & femenino \\
\hline Edad (años) & 43 & 22 & 14 & 47 & 31 & 18 & 44 \\
\hline \multirow[t]{2}{*}{ Entrenamiento musical - tipo } & si & \multirow[t]{2}{*}{ no } & si & \multirow[t]{2}{*}{ no } & \multirow[t]{2}{*}{ no } & si & \multirow[t]{2}{*}{ no } \\
\hline & básico & & básico & & & avanzado & \\
\hline Disfrute musical & si mucho & si & si mucho & si mucho & si & si mucho & si \\
\hline Umbral tono $262 *$ & 1.72 & 3.39 & 0.67 & 0.83 & 8.72 & 0.50 & 1.0 \\
\hline Umbral tono $330 *$ & 0.50 & 1.56 & 0.61 & 4.39 & 4.44 & 0.50 & 0.94 \\
\hline Umbral tono 391 * & 0.72 & 3.22 & 0.61 & 1.50 & 7.06 & 0.50 & 0.72 \\
\hline Melodia \% & 83.33 & 33.33 & 69.44 & 69.44 & 25 & 86.11 & 36.11 \\
\hline Timbre\% & 62.50 & 62.50 & 75.00 & 66.67 & 33.33 & 83.33 & 45.8 \\
\hline
\end{tabular}

* Mediana

\begin{tabular}{|c|c|c|c|c|c|}
\hline Sujetos & Sujeto 8 & Sujeto 9 & Sujeto 10 & Sujeto II & Sujeto 12 \\
\hline \multirow[t]{2}{*}{ Tipo de paciente } & \multirow[t]{2}{*}{ implantado } & \multirow[t]{2}{*}{ bimodal } & implante & implante & implante \\
\hline & & & unilateral & unilateral & unilateral \\
\hline Género & femenino & femenino & masculino & masculino & masculino \\
\hline Edad (años) & 56 & 40 & 42 & 43 & 39 \\
\hline $\begin{array}{l}\text { Tiempo de deprivacion antes del implante en } \\
\text { meses }\end{array}$ & 60 & 36 & 120 & 60 & 12 \\
\hline Tiempo de uso de su implante en meses & 12 & 6 & 4 & 108 & 180 \\
\hline Tipo de implante & Freedom & Freedom & Freedom & Medel & Nucleus 22 \\
\hline Tipo de procesador & Freedom & Freedom & Freedom & $i$ & Freedom \\
\hline Tipo de estrategia & Ace & Ace & Ace & $i$ & Speak \\
\hline Número de electrodos insertados & 18 & 22 & 21 & $i$ & 22 \\
\hline Número de electrodos activos & 11 & 22 & 21 & $i$ & 16 \\
\hline Entrenamiento musical & no & no & no & no & no \\
\hline Disfrute musical & si mucho & si & si mucho & si & si \\
\hline Umbral tono 262 & 2.17 & 6.28 & 3.61 & 4.28 & 1.67 \\
\hline Umbral tono 330 & 7.50 & 2.50 & 2.94 & 4.28 & 3.72 \\
\hline Umbral tono 391 & 8.56 & 8.56 & 5.50 & 2.61 & 4.17 \\
\hline Melodias \% & 2.78 & 13.8 & 8.33 & 19.44 & 5.56 \\
\hline Timbre \% & 4.17 & 45.83 & 50.00 & 20.83 & 33.33 \\
\hline
\end{tabular}




\section{Prueba de percepción musical con audición normal}

En la percepción del tono el resultado del umbral mediano (RI) por frecuencias fue para el tono de $262 \mathrm{Htz}$ 1 (RI 0.67 a 3.39), para $330 \mathrm{Htz} 0.94$ (RI 0.5 a 4.39 ) y para $391 \mathrm{Htz} 0.72$ (RI 0.61 a 3.22). El porcentaje de reconocimiento con las melodías presentó una mediana de $69 \%$ (RI 33,3 a 83,3\%) y el del timbre musical, medido por el reconocimiento de los instrumentos fue de $62,5 \%$ (RI 45,8 a 75\%). La duración mediana total fue de 25.85 minutos. Para la prueba de tonos fue de 9.21 , 12.49 en la de melodías y 4.52 en la de timbre musical.

\section{Familiaridad previa con la prueba de melodías en ambos grupos}

El reconocimiento de melodías en ambos grupos y en especial en los implantados fue señalado como la prueba más difícil. Por esta razón, el grupo de investigación quiso determinar si la familiaridad previa con la melodía tenía alguna repercusión en la facilidad para reconocerla en la prueba. Para la canción happy birthday, que era conocida para el $100 \%$ de los evaluados, se encontró que el 50\% no fue capaz de reconocerla y solo dos de los doce lo logró las tres veces que se les presentó. En Here comes de bride (marcha nupcial) conocida por todos los participantes, los aciertos con la melodía aumentan, pero tampoco son de $100 \%$. La canción con menor familiaridad fue Three blind mice, solo conocida por uno de los evaluados $(8,3 \%)$, se encontró que cinco no la identificaron ni una sola vez y dos lo hicieron tres veces.

\section{Discusión}

El concepto de música al ser universal, en principio es una ventaja para la prueba, pero se vio un poco truncada al usar algunas canciones no tan populares en nuestro medio de habla hispana en esta población piloto. Por otro lado, revisando el estudio que estandarizó la prueba nos alentaba ver cómo las canciones más comunes como Happy birthday o Here comes the bride, no eran las más identificadas por los sujetos evaluados por el estudio de Kang y colaboradores. ${ }^{16} \mathrm{El} 50 \%$ de los participantes no identificaron ni una vez el Happy birthday y $33 \%$ no reconoció la marcha nupcial. Lo que cuestiona la importancia de la familiaridad previa con las melodías, pudiendo ser más relevante qué tan reconocible es la melodía después de la cancelación del ritmo musical a la que se someten en el CAMP test. El hecho de no conocer las melodías podría en parte subsanarse con el entrenamiento previo a la prueba donde se presentan dos veces cada canción.

\section{Cambios en el formulario de características demográficas y percepción musical}

Al valorar el cuestionario de conocimiento musical, la pregunta iha tenido entrenamiento musical previo a la sordera? amerita ser modificada debido a que será aplicada también a individuos con audición normal. En la misma sección se indaga sobre el disfrute musical con la pregunta ¿yo me describiría como una persona que disfruta la música? y sus opciones de respuesta son: 1) muy en desacuerdo, 2) en desacuerdo, 3) de acuerdo y 4) muy de acuerdo. Los investigadores consideramos que podría cambiarse por: respecto a disfrutar la música diría que: 1) no me gusta para nada, 2) no me gusta, 3) me gusta y 4) me gusta muchísimo. En la prueba piloto la pregunta no era de fácil comprensión para todos los participantes.

Otro dato clínico, adicional al tipo de implante utilizado, que debe incluirse es la estrategia de programación utilizada en cada paciente, pues dependiendo de los electrodos estimulados, la velocidad y la combinación de los mismos, puede ser una variable que influya en los resultados de la percepción musical. En cuanto al número de electrodos, es importante conocer tanto los insertados como los activados. Se incluirá un apartado en el que se indague sobre la familiaridad de las canciones evaluadas con el fin de ver la relación entre este dato y los aciertos con las melodías, pues no se había considerado y durante la prueba pudo ser una variable de relevancia en la percepción musical. Por lo anterior, el apartado cuatro pasará a ser el punto cinco. 


\section{Observaciones a la prueba musical CAMP}

En todos los pacientes se percibió no solo la necesidad de concentración, sino además un importante componente de atención y memoria por tiempo prolongado. Estas características no son medibles en forma directa durante la prueba, hallazgo señalado por Gfeller en su estudio publicado en $2008^{19}$ donde demuestra que las pruebas cognitivas apuntan al uso de áreas especializadas de procesamiento y memoria, de gran importancia para el máximo beneficio obtenido a través de los implantes existentes en el momento. Para todos los individuos el test catalogado como más difícil fue el de melodías, en especial en la población con IC ( 5 de 5) y en seis de siete normales.

En todos los componentes de percepción musical, tono, melodía y timbre, los resultados fueron mejores para los sujetos normales. Mientras los procesadores de implante (en los cuales es típica la extracción de la cubierta temporal) pueden transmitir los patrones de tiempo con alta fidelidad, la información del espectro, como aquellas necesarias para el tono, se transmiten en forma degradada. Debido a que las relaciones de los tonos entre una serie de notas (incluyendo el contorno, dirección y cambio en el tono y la distancia de los intervalos) proveen la base de las melodías, es esta una de las características más difíciles para los pacientes con IC. ${ }^{2}$

Hubo valores mayores del umbral de detección de la diferencia tonal para las tres frecuencias evaluadas; sin embargo, se observó alguna sobreposición de los resultados individuales en especial en los 262 y 330 Htz. Los altos umbrales encontrados en los pacientes implantados para la percepción del cambio en los tonos para todas las frecuencias evaluadas de 262 , 330 y $391 \mathrm{Htz}$ concuerdan con el concepto ya sabido que para los pacientes implantados es necesario más de un semitono (de cuatro a ocho semitonos) para poder distinguir que son dos los tonos que suenan y la dirección del incremento de los mismos. ${ }^{2,4,20}$ Para el timbre y la melodía la ventaja de aquellos con audición normal fue marcada y clara en especial para la última.
El amplio rango de los resultados hace más interesante el análisis de la mediana como medida de tendencia central. La variabilidad en las respuestas, en especial si observamos las de los implantados es evidente no solo entre los sujetos sino también en el mismo individuo según la característica musical evaluada. Esto indica, por ejemplo, que una mala puntuación en la prueba de melodías, no implicó un mal puntaje en la misma proporción en la de timbre musical (S9, S10). Encontramos además, que el buen desempeño en la detección de tonos no siempre refleja una adecuada percepción de melodías (paciente S 12, concordando con las observaciones del estudio de Kang). ${ }^{16}$

La familiaridad con la melodía no siempre arroja los resultados lógicos esperados de mayores aciertos, como vimos en el caso de Happy birthday o Here comes the bride, observaciones ya reportadas. ${ }^{16} \mathrm{El}$ timbre musical es una característica del sonido cuyos atributos más fuertes son la envoltura espectral y temporal, que pueden variar según el instrumento tocado e incluso en el mismo dependiendo del tono, la intensidad y la expresión musical de una nota..$^{2 n, 21}$

En nuestros resultados es evidente la ventaja de los normoyentes en la distinción entre los diferentes instrumentos, siendo notoria con trompeta, flauta, guitarra y piano en orden de importancia. En el grupo de implantados los mejores resultados se obtuvieron con guitarra, saxofón y piano, en dicho orden.

\section{Entrenamiento musical previo}

Se hace especial mención que las mejores medianas se encontraron en el único sujeto con entrenamiento musical formal previo con guitarra. El obtuvo para umbral de percepción de tonos en $262 \mathrm{Htz}$ : 0.5 (error estándar (EE) de 0), en $330 \mathrm{Htz} 0.5$ (EE:0), $391 \mathrm{Htz}$ (EE:0), es decir era necesario menos de un semitono para percibir la diferencia entre las notas. Sus resultados en la percepción de melodía fue $86 \%$ y timbre $83 \%$. Nuestros resultados concuerdan con lo encontrado por Gfeller en 2008 , en donde un factor predictivo positivo en el desempeño musical es el entrenamiento previo recibido en este campo. ${ }^{19}$ Estos datos nos hacen pensar que sería interesante en el estudio de correlaciones de 
habla y percepción musical tener un número mayor de personas con entrenamiento musical previo, para ver cómo se comporta este subgrupo.

\section{Conclusiones}

La prueba $C A M P$ de percepción musical resulta útil y fácil de ser autoadministrada tanto en normoyentes como en usuarios de IC cuyo idioma nativo sea español, al suministrar las cartillas de traducción de los comandos. La mayor dificultad encontrada fue con la prueba de melodías, sobre todo en los pacientes implantados. La falta de familiaridad con las melodías para los sujetos con idioma nativo español y su influencia en los resultados de reconocimiento de las mismas debe estudiarse en una población mayor para saber si conviene tenerla en cuenta o solo basarnos en las pruebas de tonos y timbre que tienen una distribución más uniforme.

\section{Referencias}

1. Peñaranda A, García JM, García J, Baron C. The experience in Colombia South America with Nucleus 22 channel cochlear implants. Adv Otorhinolaryngol. 1997;52(312):314

2. Gfeller K, Tumer C, Oleson J, Zhang X, Gantz B, Froman R, et al. Accuracy of cochlear implant recipients on pitch perception, melody recognition and speech and noise. Ear Hear. 2007;28(3):412-28

3. Lalwani AK, Larky JB, Wareing MJ, Kwast K, Schindler RA. The Clarion Multi Strategy Cochlear Implant-Surgical Technique, Complications, and Results: A Single Institutional Experience. Otology \& Neurotology. 1998;19(1):66.

4. Gfeller K, Witt S, Woodworth G, Mehr MA, Knutson J. Effects of frequency, in strumental family, and cochlear implant type on timbre recognition and appraisal. The Annals of Otology, Rhinology, and Laryngology. 2002;111(4):349

5. Limb CJ. Structural and functional neural correlates of music perception. Anatomical Record Part A Discoveries in Molecular Cellular and Evolutionary Biology. 2006;288(4):435.
6. Lassaletta L, Castro A, Bastarrica M, Pérez-Mora R, Herrbn B, Sánz L, et al. Pe cepción y disfrute de la música en pacientes poslocutivos con implante coclear Acta Otorrinolaringológica Española. 2008;59(5):228-34.

7. Zatorre RJ, Belin P. Spectral and temporal processing in human auditory cortex Cereb Cortex. 2001;11:946-53.

8. Samson S, Zatorre RJ, Ramsay JO. Deficits of musical timbre perceptions after unilateral temporal-lobe lesion revealed with multidimensional scaling. Brain 2002;125:511-23

9. Liegeois-Chauvel C, Peretz I, Babai M, Laguitton V, Chauvel P. Contribution of different cortical areas in the temporal lobes to music processing. Brain. 1998;121:1853-67.

10. Lee DJ, Chen Y, Schlaug G. Corpus callosum: musician and gender effects. Neu roreport. 2003;14:205-9.

11. Schlaug G, Jancke L, Huang Y, Steinmetz H. In vivo evidence of structural brain asymmetry in musicians. Science. 1995;267:699-701.

12. Drennan WR, Rubinstein JT. Music perception in cochlear implant users and its relationship with psychophysical capabilities. J Rehabil Res Dev. 2008;45(5):779-89.

13. McDermott HJ. Music perception with cochlear implants: a review. Trends in Amplification. 2004;8(2):49.

14. Pijl S. Musical pitch perception with pulsatile stimulation of single electrodes in patients implanted with the nucleus cochlear implant. The Annals of Otology, Rhinology \& Laryngology Supplement. 1995; 166:224.

15. Kong YY, Cruz R, Jones JA, Zeng FG. Music perception with temporal cues in acoustic and electric hearing. Ear and hearing. 2004;25(2):173.

16. Kang R, Nimmons GL, Drennan W, Longnion J, Ruffin C, Nie K, et al. Devel opment and Validation of the University of Washington Clinical Assessment of music perception test. Ear and hearing. 2009;30(4):411-8.

17. Nimmons GL, Kang RS, Drennan WR, Longnion J, Ruffin C, Worman T, et al Clinical assessment of music perception in cochlear implant listeners. Otology \& Neurotology: official publication of the American Otological Society, American Neurotology Society [and] European Academy of Otology and Neurotology. 2008;29(2):149.

18. Li XC, Friedman RA. Nonsyndromic hereditary hearing loss. Otolaryngol Clin North Am. 2002 Apr;35(2):275-85.

19. Gfeller K, Oleson J, Knutson J, Breheny P, Driscoll VD, Olzewski C. Multivaria te predictors of music perception and appraisal by adult cochlear implant users. J Am Acad Audiol. 2008;19:120-34.

20. Driscoll VD, Oleson J, Jiang D, Gfeller K. Effect of instrument timbre on melodic contour identification by cochlear implant users. J Am Acad Audiol. 2009;20:71-82

21. Galvin JJ, Fu QJ, Oba S. Effect of instrument timbre on melodic contour identification by cochlear implant users. J Acoust Soc Am. 2008;124(4):189-95. 